\title{
A Critical Review on the Rationality of Popular Failure Criteria for Composites
}

\author{
Shuguang Li and Elena Sitnikova \\ Faculty of Engineering, University of Nottingham, University Park, Nottingham NG7 2RD, UK
}

\begin{abstract}
Popular failure criteria for fibre reinforced composites are subjected to critical scrutiny on their rationality. The rationality of a theory is about the mathematical and physical logic underlying the theory, rather than the closeness of their results in comparison with experimental data. Seeking for close comparisons with a set of experimental data before obtaining a basic level of rationality is not a scientific attitude, although the practice is becoming the norm in the community of science and engineering. A theory lacking of rationality can never be a sound one no matter how closely it compares with experimental data in one respect or another, since a good comparison in one respect comes at a price of poor agreement in some other respects usually, either unknown for the time being or hidden deliberately. The objective of this paper to raise the awareness of rationality, or the lack of it, in existing theories so that the users will be warned to exercise their judgement on the applicability of these theories before employing them in future. It should also help the researchers avoid incorporating illogical considerations into the formulations of the new theories they are developing.
\end{abstract}

\section{$1 \quad$ Introduction}

How composites fail under loading has been a key question to answer from day one of serious applications of these materials, and the emergence of various failure criteria reflected such needs. Some of the criteria have been routinely publicised, e.g. through textbooks, and widely employed, e.g. in commercial analysis and design codes. However, serious engineering practices seem to present a rather different picture, in particular, in the aerospace industry, where considerations are mainly based on the so-called design allowables [1]. There are two basic categories of such design allowables, one at materials level based on coupon tests and the other on the structural level ranging from typical laminate layups to various degrees of sophistication with features, such as notches, holes, joints, etc. and scales, such as parts, subcomponents, components and complete structures in a so-called 'building block approach' [2]. Theoretical failure criteria have largely been by-passed in such an approach, given the efforts made to the development of various failure criteria. The root 
reason, said or unsaid, has been the simple fact that the theoretical criteria do not seem to offer useful enough guidance to the actual design practices. In response to the complaint of lack of accuracy of existing theories, theoreticians have been showing their determination to resolve the problem by escalating their levels of sophistication, often, coming with more unsupported assumptions or undeterminable (in terms of existing testing standards) material properties. As a result, instead of bringing direct solutions to engineering practitioners, new theories tend to drive them further away, sometimes, to such an extent that they could not be bothered by those theories anymore but plunged back to their exercise of determining the design allowables. The endeavours seem to bifurcate widely and deeply.

It is fair to say that the design allowables, once available, are simple and safe to use. There have been established procedures to follow in engineering [1]. However, the shortcoming of this approach is that the process of obtaining a sufficient set of design allowables for a given material is very demanding, as it is both labour intensive and time-consuming, in addition to high material costs. Practical affordability would restrict the scope to a limited number of materials, layups, geometric dimensions, etc. More critically, it makes the process of adopting any new material a formidable task. It is certainly not a comfortable position a creative designer would like to find $\mathrm{him} /$ herself in. However, there does not seem to be any alternatives.

It is worth noting that practical failures of composites often involve delamination due to impact. Delamination mechanisms are usually associated with the structural behaviour, rather than the material failure that conventional failure criteria aim to address. This indicates that there is a significant gap between the existing failure criteria and engineering practices. One might argue that there are a lot of attempts to understand impact and delamination. However, if one is honest and also really knows what he/she is saying, the truth would be that the existing understanding with reasonable reliability on this subject is very limited. Examples have been shown in a recent paper [3] demonstrating that, even for the simplest problem of this kind, lack of understanding could be easily identified, let alone for any more sophisticated scenarios.

Without discouraging theoreticians completely, one would probably agree that there is a substantial set of problems where failure is dictated by ideal mechanisms of failure of materials as opposed to structures. For these problems, can the state-of-the-art failure criteria have high enough fidelity in capturing the failure? Surely, one would like to have a categorically positive answer to this question. However, to practitioners' dismay, an honest answer would likely to be 'Not sure'. This will be the focal point of the present paper, before one is in any credible position to address more sophisticated problems, such as impact and delamination. 
The series of World Wide Failure Exercises [4,5,6] have made significant contribution to the clarification of the position, where a wide range of popular theories have been appraised primarily based on comparisons with a large, but still limited, number of experimental data. On the theoretical side, those involved in theoretical developments probably have no shortage of occasions when correct results were accidentally obtained from wrong theories. On the other hand, without undermining the role of experiments, an honest and critical experimentalist probably would agree that there could be just as many chances to obtain wrong results in experiments as in theoretical work. The assessments achieved solely by comparisons with experimental data are bound to be of limited authority on the fidelity of any specific criterion.

There is lack of systematic reviews on the rationality of failure theories. It is the intention of the present paper to make an attempt along this line. By disclosing the irrational aspects embedded in existing popular failure criteria, the aim of this paper is to ring a loud alarm bell for a sober reflection of the state-of-the-art before meaningful efforts can be channelled to the genuine front line to solve engineering problems.

To facilitate the discussion in this paper, attention will be paid only to the class of composites exhibiting transverse isotropy. Practically, the applicability of all existing failure criteria has been limited to this class of materials. Although some criteria presented themselves as if having wider applicability, their meaningful applications always narrow down to transversely isotropic materials. For instance, the Tsai-Wu criterion was initially proposed for orthotropic materials in general as far as its formality is concerned before being specialised to transversely isotropic materials. However, any attempt of applying it to an orthotropic material, e.g. a quasi-isotropic laminate, hardly produces any results bearing relevance to reality. In addition, regarding other considerations, such as thermoset or thermoplastic, with toughened matrix or untoughened, with strong bonding between fibre and matrix or weak bonding, high strength or high modulus fibres, glass or carbon fibres, etc. all theories under review in this paper fall in the category of phenomenological approaches. The spirit of such approaches is that they apply to all systems. Any difference in the composite systems should be duly reflected in the strength properties employed in the criteria. Whilst this reveals the shortcomings of phenomenological approaches in general, it offers attraction to design practitioners.

For the clarity of the present paper, a rational theory is defined as one that is based on wellestablished physical rules (often, common sense), e.g. the objectivity, and a definitive number of independent assumptions, e.g. the existence of a failure envelope. These assumptions should be sufficient (even better, if also necessary) for the theory. They should not compromise the physical 
rules and not be self-contradictive, explicitly or implicitly. The theory should be deduced from the physical rules and the assumptions free from any logical fallacy.

It is certain that no one sets off to produce an irrational theory. However, this alone does not prevent irrational theories from being produced. Theoreticians are supposed to bear the rationality requirements as stated above in mind during the development of their theories but the reality is that not all theories available were produced by such theoreticians. Without these requirements tightly fastened in their minds, theory developers are likely to overlook one aspect or another. It is usually far more difficult to iron any irrational elements out than putting them in, as before any of them can be ironed out, its presence has to be appreciated first, which defines the purpose of the present paper for a range of popular failure criteria. It may be true that some of the points made in this paper had been realised by other researchers in the past. As they are not found in the open literature, to the best of the authors' knowledge, they deserve to be made available to a wide community to benefit other researchers and practitioners.

\section{The maximum stress criterion}

The maximum stress criterion is definitely one of the most popular criteria in use. It can be presented as

$\frac{\sigma_{i}}{\sigma_{i t}^{*}} \leq 1 \quad$ if $\sigma_{i} \geq 0 \quad$ or $\quad \frac{\left|\sigma_{i}\right|}{\sigma_{i c}^{*}} \leq 1 \quad$ if $\sigma_{i}<0 \quad(i=1,2$ and 3$)$

$\frac{\left|\tau_{j}\right|}{\tau_{j}^{*}} \leq 1 \quad(j=23,13$ or 12$)$

where $\left\{\begin{array}{llllll}\sigma_{1} & \sigma_{2} & \sigma_{3} & \tau_{23} & \tau_{13} & \tau_{12}\end{array}\right\}$ defines the stress state in the material's principal axes, $\sigma_{1 t}^{*}$ and $\sigma_{1 c}^{*}$ are the tensile and compressive strengths in the fibre direction, $\sigma_{2 t}^{*}=\sigma_{3 t}^{*}$ and $\sigma_{2 c}^{*}=\sigma_{3 c}^{*}$ the tensile and compressive strengths transverse to the fibres, and $\tau_{23}^{*}$ and $\tau_{13}^{*}=\tau_{12}^{*}$ the transverse and longitudinal shear strengths.

Apparently, the title of the criterion is not meant to be understood literally. It is not the maximum stress but the maximum stress ratio that counts. This criterion is so traditional that its origin can hardly be traced. It is perhaps a good thing, as it is the glitch in the criterion that this paper is to reveal, hence it would not be considered as any individual's fault. If anything, it should be the collective failure of the community for having overlooked basics of this criterion.

The weakness of the maximum stress criterion has been commonly pointed out as the lack of interactions between different stress components. Many subsequent developments tended to 
incorporate interactions as will be reviewed later in this paper. Assuming perfectly measured strength properties employed in constructing the failure envelope, one can place absolute confidence only on the predictions at the intersections between the envelope and the coordinate axes, as these are the test data experimentally measured directly.

There is a more fundamental deficiency which does seem to have been overlooked by large. It is the lack of objectivity. Objectivity is a basic rule of physics and, in fact, science in general, which requires that the consequence of any physical process not vary with the coordinate system, i.e. the perspective of the observer, employed to describe the physical process. To reveal the aspect lacking objectivity in the maximum stress criterion, consider a 2D equal tensile and compressive biaxial stress state in the plane transverse to the fibres in a UD composite which is usually regarded as a transversely isotropic material. The application of the criterion predicts a tensile failure at the tensile strength, $\sigma_{2 t}^{*}$, due to the tensile stress component, as most composites are typically brittle and hence have lower tensile strength than the compressive one. The failure mode would be in the way as depicted in Figure 1(a). However, if one views the same stress state at $45^{\circ}$ off the axis, it is in pure shear as shown in Figure 1(b) with $\tau=\sigma$. Application of the same criterion would result in prediction of the failure at the transverse shear strength, $\tau_{23}^{*}$, with a fracture surface likely being on the action plane of the shear stress (marked by the dashed line in Figure 1(b)). It has been shown now that different results are obtained for the same problem simply due to the fact that the same physical process has been observed from two different perspectives with reference to two coordinate systems between (a) and (b) in Figure 1. This is an apparent violation of the objectivity rule of physics.

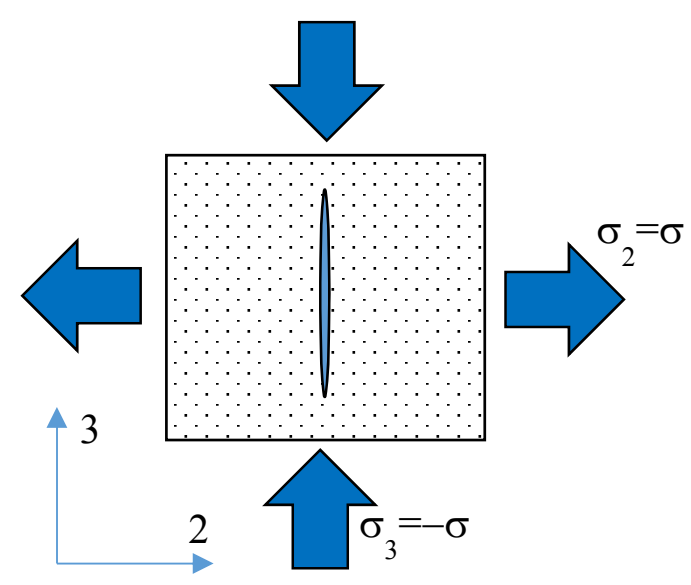

(a)

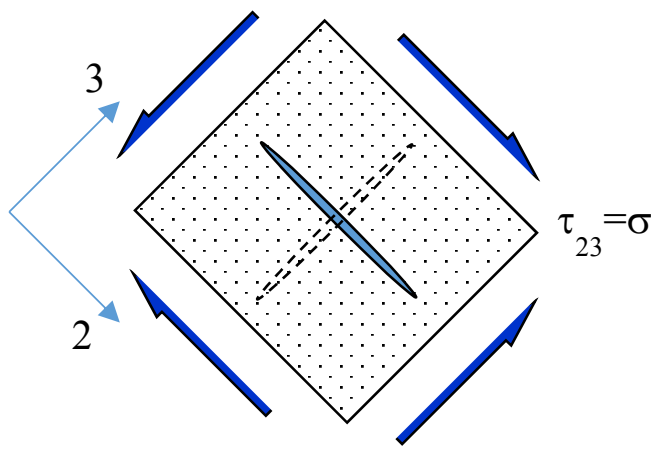

(b)

Figure 1 Equivalent stress states giving different strengths and failure modes due to different viewpoints 
One might argue that failure under pure shear is expected at $45^{\circ}$ to the action plane of the shear stress. However, this results from the use of the maximum principal stress criterion [7] for isotropic materials (UD composites are isotropic in their transverse plane to fibres) independent of the maximum stress criterion concerned here for composites. This cannot be obtained from the original maximum stress criterion alone. Even so, accepting this extra consideration, one would immediately have $\tau_{23}^{*}=\sigma_{2 t}^{*}$ as a natural consequence. Whilst this would be a nice position to be in, since one strength property fewer needs to be measured, in particular given the fact that the measurement of $\tau_{23}^{*}$ is usually a lot more demanding than that of $\sigma_{2 t}^{*}$, it is not what one always observe practically between these two properties.

The point of the elaboration above is to reveal an irrational aspect of the maximum stress criterion, $v i z$. the lack of objectivity. It is more fundamental than the lack of interactions between stresses and yet it has never been paid any attention to in the literature to the best of the authors' knowledge. Exposing the flaws in the very foundations of one of the most basic and common composites failure criteria vividly demonstrates the necessity of scrutinising the foundations of any theoretical framework before building much sophistication on top of that.

\section{$3 \quad$ The maximum strain criterion}

Superficially, the maximum strain criterion can be viewed as a straightforward counterpart of the maximum stress criterion with corresponding terms being the strains rather than the stresses, as follows.

$\frac{\varepsilon_{i}}{\varepsilon_{i t}^{*}} \leq 1 \quad$ if $\varepsilon_{i} \geq 0 \quad$ or $\quad \frac{\left|\varepsilon_{i}\right|}{\varepsilon_{i c}^{*}} \leq 1 \quad$ if $\varepsilon_{i}<0 \quad(i=1,2$ and 3$)$

$\frac{\left|\gamma_{j}\right|}{\gamma_{j}^{*}} \leq 1 \quad(j=23,13$ or 12$)$

where $\left\{\begin{array}{llllll}\varepsilon_{1} & \varepsilon_{2} & \varepsilon_{3} & \gamma_{23} & \gamma_{13} & \gamma_{12}\end{array}\right\}$ defines the strain state in the material's principal axes, $\varepsilon_{1 t}^{*}$ and $\varepsilon_{1 c}^{*}$ are the tensile and compressive failure strains in the fibre direction, $\varepsilon_{2 t}^{*}=\varepsilon_{3 t}^{*}$ and $\varepsilon_{2 c}^{*}=\varepsilon_{2 t}^{*}$ are the tensile and compressive failure strains transverse to fibres, and $\gamma_{23}^{*}$ and $\gamma_{13}^{*}=\gamma_{12}^{*}$ are the transverse and longitudinal shear failure strains. It should be noted that all the direct strains at failure have to be measured under corresponding uniaxial stress states and the shear strains at failure under corresponding pure shear stress states, according to any available standard. Readers are gently reminded that uniaxial strain states and pure shear strain states are unachievable 
practically, in particular, if one wishes avoid premature failure of the material due to effects such as stress concentration.

When assessing the failure of the material, the choice of a stress or strain based criterion may be perceived as a matter of preference. Because of the disparity in the choices, one would not expect the identical predictions from them as a result. In fact, it can often be found in the literature that the differences are shown through an illustration as given in Figure 2, by plotting in the stress plane the failure envelopes from each of them for the same material under in-plane stress states involving direct stresses only.

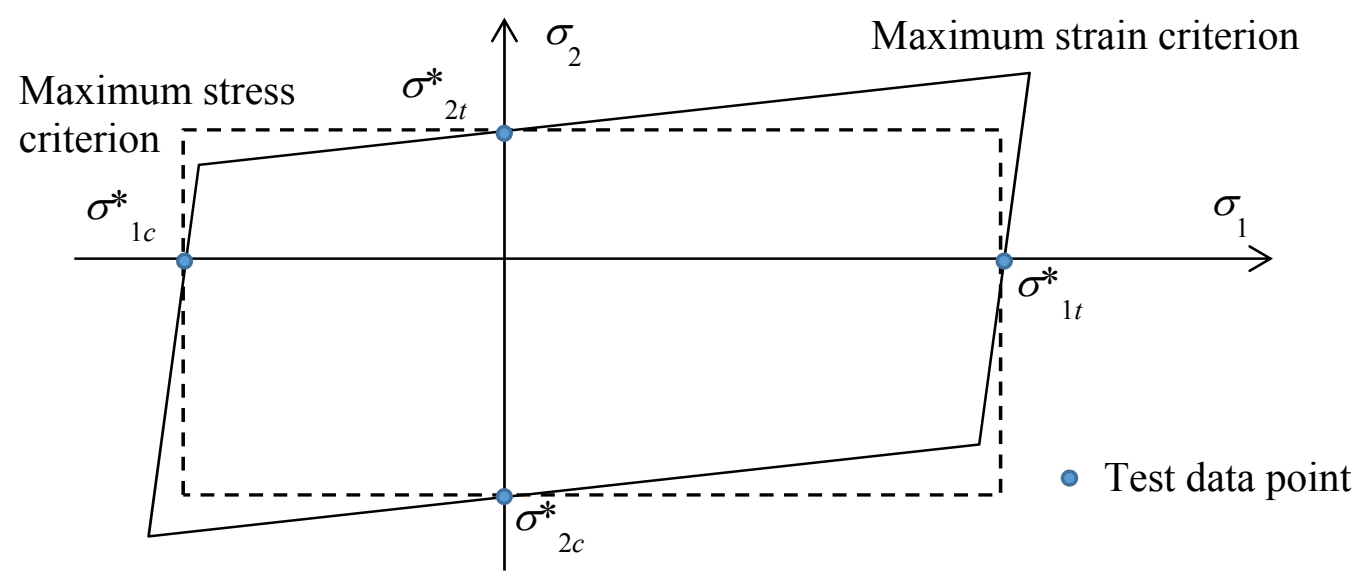

Figure 2 Failure envelopes in the stress plane from the maximum stress and maximum strain criteria, respectively

However, it might be more revealing if one plots the same in the strain plane instead. Due to the commonality of uniaxial stress states employed, the failure strains can be obtained from the failure stresses as

$\varepsilon_{1}^{*}=\sigma_{1}^{*} / E_{1}, \quad \varepsilon_{2}^{*}=\sigma_{2}^{*} / E_{2}$

where $\varepsilon_{1}^{*}=\left\{\begin{array}{lll}\varepsilon_{1 t}^{*}, & \text { if } & \sigma_{1}^{*}=\sigma_{1 t}^{*} \\ \varepsilon_{1 c}^{*} & \text { if } & \sigma_{1}^{*}=\sigma_{1 c}^{*}\end{array}\right.$ and $\varepsilon_{2}^{*}=\left\{\begin{array}{lll}\varepsilon_{2 t}^{*}, & \text { if } & \sigma_{2}^{*}=\sigma_{2 t}^{*} \\ \varepsilon_{2 c}^{*} & \text { if } & \sigma_{2}^{*}=\sigma_{2 c}^{*}\end{array}\right.$

In order to plot the maximum stress failure envelope to the strain plane, consider biaxial stress states,

$\varepsilon_{1}=\left(\sigma_{1}-v_{12} \sigma_{2}\right) / E_{1}$

$\varepsilon_{2}=\left(\sigma_{2}-v_{21} \sigma_{1}\right) / E_{2}$

The four corners can be determined by allowing the stresses to take four possible combinations of the strengths, 


$$
\begin{aligned}
& \left(\sigma_{1}^{*}=-\sigma_{1 c}^{*} \quad \text { and } \quad \sigma_{2}^{*}=\sigma_{2 t}^{*}\right) \quad\left(\sigma_{1}^{*}=\sigma_{1 t}^{*} \quad \text { and } \quad \sigma_{2}^{*}=\sigma_{2 t}^{*}\right) \\
& \left(\sigma_{1}^{*}=-\sigma_{1 c}^{*} \quad \text { and } \sigma_{2}^{*}=-\sigma_{2 c}^{*}\right) \quad\left(\sigma_{1}^{*}=\sigma_{1 t}^{*} \quad \text { and } \quad \sigma_{2}^{*}=-\sigma_{2 c}^{*}\right) \text {. }
\end{aligned}
$$

The maximum stress failure envelope can then be plotted in the strain plane as shown in Figure 3 .

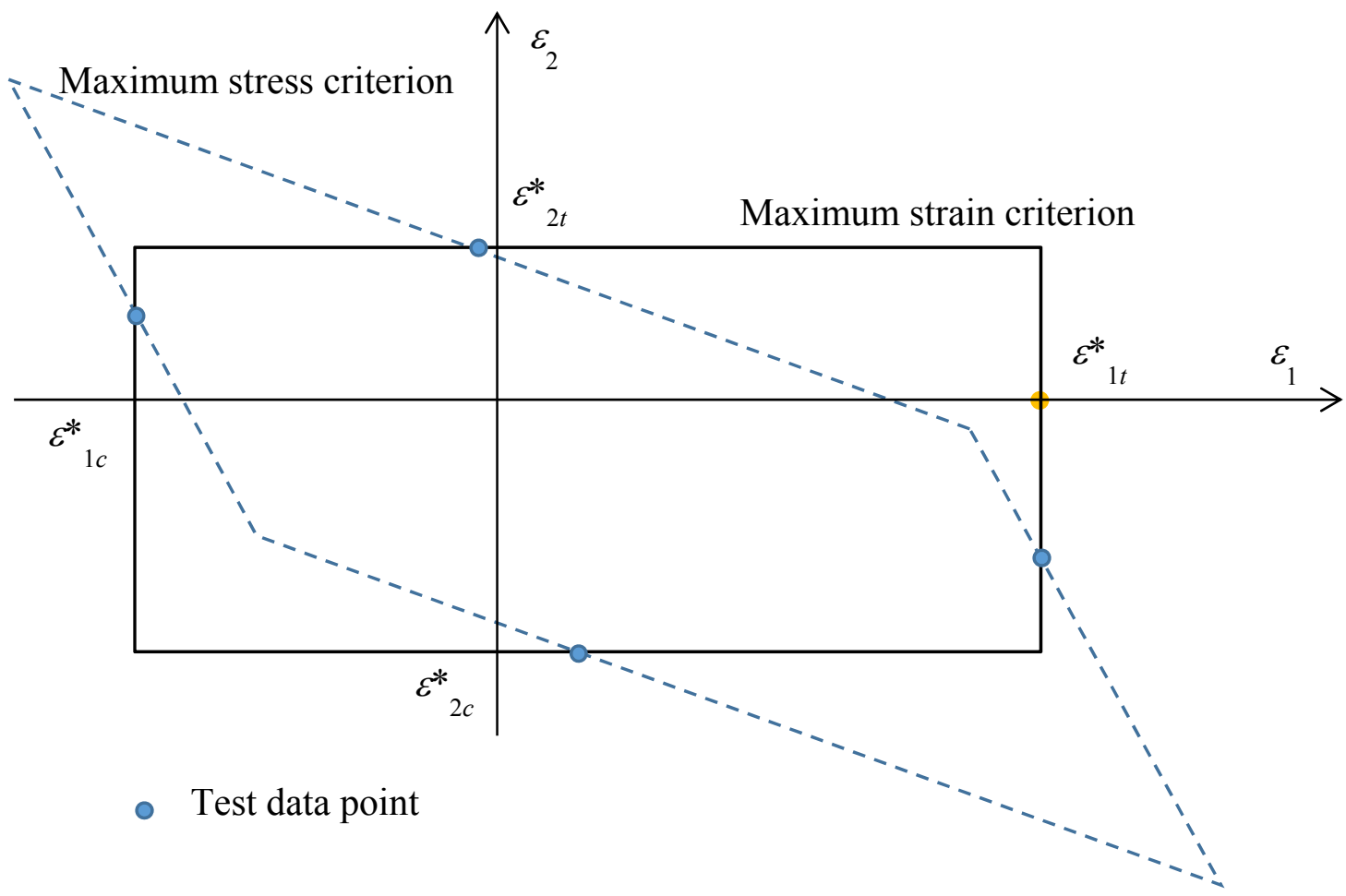

Figure 3 Failure envelopes in the strain plane from the maximum stress and maximum strain criteria, respectively.

The following observations and comments can be made.

1) The two envelopes do not intersect at coordinate axes anymore as they did in Figure 2 . The reason is that the testing data are never found on the coordinate axes, as they would correspond to uniaxial strain states, which can hardly be materialised in modern laboratory conditions. The bottom line is that this is no industrial standard available to support such measurements. Experimental data have to be obtained under the uniaxial stress states, not the uniaxial strain state.

2) Because of the explanation given above, failure predictions made using the maximum strain criterion will be reasonable only around the points of intersection of the two envelopes, since these are the points that are actually obtained experimentally.

3) When Figure 2 is produced, a plane stress condition is implied and this is usually what readers would perceive intuitively without enquiring. However, for Figure 3, unless it has been specified, readers would not be able to tell if the stress states involved in the failure envelopes correspond to a plane stress condition $\left(\sigma_{3}=0\right)$ or a plane strain condition $\left(\varepsilon_{3}=0\right)$. 
Some could not even be bothered with the differences in between. Practically, a plane stress condition is a lot simpler to obtain in this case. To clarify the position, Figure 3 should be understood as under the plane stress condition.

One of the advantages of the non-interactive criteria, such as the maximum stress or strain, is that they offer a prediction of the failure mode, in addition to their simplicity. Whilst the failure mode predicted by the maximum stress criterion enjoys much success, except the irrational aspect as revealed in the previous section, the prediction from the maximum strain criterion could be rather misleading. Due to the effects of Poisson's ratios, a tensile stress in the transverse direction can be accompanied by zero or even negative strain in the same direction. Specifically, according to the maximum strain criterion, at the point as marked by a yellow blob in Figure 3 the failure should be in the fibre direction giving a fracture surface transverse to fibres, since there are no strains in other directions. However, if one has any faith in the maximum stress criterion, the stress ratios obtained would suggest that the transverse tension is more critical than the fibre direction tension. For instance, consider a T300 carbon/epoxy composite of the following material properties.

$$
\begin{array}{lll}
E_{1}=138 \mathrm{GPa}, & E_{2}=11 \mathrm{GPa}, & v_{12}=0.28, \\
\sigma_{1 t}^{*}=1500 \mathrm{MPa}, & \sigma_{1 c}^{*}=900 \mathrm{MPa}, & \sigma_{2 t}^{*}=27 \mathrm{MPa}, \quad \sigma_{2 c}^{*}=200 \mathrm{MPa}
\end{array}
$$

The point marked in Figure 3 corresponds to a strain state with a vanishing strain in the transverse direction of the material and the critical longitudinal strain value is

$\varepsilon_{1 t}^{*}=\sigma_{1 t}^{*} / E_{1}=1.087 \%$

At $\varepsilon_{1}=\varepsilon_{1 t}^{*}$, the stresses can be found as follows.

$$
\begin{aligned}
& \sigma_{1}=\frac{E_{1}}{1-v_{12} v_{21}} \varepsilon_{1 t}^{*}=1410 \mathrm{MPa}=0.94 \sigma_{1 t}^{*}<\sigma_{1 t}^{*} \\
& \sigma_{2}=\frac{v_{21} E_{1}}{1-v_{12} v_{21}} \varepsilon_{1 t}^{*}=31.5 \mathrm{MPa}=1.17 \sigma_{2 t}^{*}>\sigma_{2 t}^{*}
\end{aligned}
$$

This indicates that according to the maximum stress criterion, transverse failure is a more likely failure scenario than the fibre failure. Using the maximum strain criterion alone, transverse failure is simply out of the question.

One can always trivialise the dispute by pointing to the fact that the stress state represented by the yellow blob in Figure 3 involves significant interactions between stress components, undermining the applicability of the maximum stress criterion. This is exactly a strong point to be made. There is little interaction between strain components at that point but it does not make the maximum strain criterion much reliable. Whether one prefers the maximum stress or strain criterion, the reliability 
and hence applicability will have to be based on the condition of the absence of strong interactions between the stresses, NOT the strains, that is common between both criteria.

There are claims that design based on strain allowables is more reliable. Whilst this could remain largely true if one restricts to his/her practices to the failure in the fibre direction, it should not be blindly generalised as it is not meant to be universally reliable, in particular, not so in the transverse direction as illustrated above. Given the comments in the previous paragraph, one probably ought to consider whether more faith has been placed on strains than they deserve. The concept of stress was invented by our ancestors for a good reason!

Some design practitioners claimed that their designs were made entirely based on strains without having to evaluate stresses. The authors would argue that these practitioners are either misled by others or are misleading others, because without stresses being evaluated, one cannot even tell what a uniaxial stress state is, whilst all available standards for measuring materials strength properties are all based on uniaxial stress states. Reader are reminded again that a uniaxial strain state should never be perceived as equivalent replacement for the uniaxial stress state. In terms of applicability of the maximum strain criterion, the condition is the absence of strong interactions between stress components, which cannot be assessed without stresses being evaluated.

\section{The Tsai-Wu criterion}

The Tsai-Wu criterion is one of the most popular criteria where full interactions between stress components are present. For transversely isotropic materials, it can be expressed as

$$
\begin{aligned}
F & =F_{11} \sigma_{1}^{2}+F_{22}\left(\sigma_{2}^{2}+\sigma_{3}^{2}\right)+\left(2 F_{22}-F_{44}\right) \sigma_{2} \sigma_{3}+2 F_{12} \sigma_{1}\left(\sigma_{3}+\sigma_{2}\right) \\
& +F_{1} \sigma_{1}+F_{2} \sigma_{2}+F_{2} \sigma_{3}+F_{44} \tau_{23}^{2}+F_{66} \tau_{13}^{2}+F_{66} \tau_{12}^{2}
\end{aligned}
$$

Tsai has modestly claimed that the criterion is an empirical one, i.e. it was devised to fit the experimental data. However, in reality, there are many rational considerations built in the formulation of the criterion, e.g. the objectivity as discussed in Section 2 when applied to transversely isotropic materials. There is indeed one empirical aspect of it, i.e. the determination of the coefficient $F_{12}$. This empiricism has only been removed recently after a rational consideration has been given to it [8]. Without reproducing the derivations there, one inconsistent aspect of the conventional Tsai-Wu criterion can be revealed as follows. 
Tsai-Wu criterion was formulated under the consideration of closed failure envelope in the stress space. However, by simply considering the biaxial direct stress state in the plane transverse to the fibres, the failure criterion reduces to [8]

$F_{22} \sigma_{2}^{2}+F_{22} \sigma_{3}^{2}+\left(2 F_{22}-F_{44}\right) \sigma_{2} \sigma_{3}+F_{2} \sigma_{2}+F_{2} \sigma_{3}=1$

If it is considered as a conic section as an intersection between the failure envelope in the 6dimensional stress space and the $\sigma_{2}-\sigma_{3}$ plane, the rules of analytic geometry apply. Depending on the value of the discriminant

$D=F_{22}^{2}-\frac{1}{4}\left(2 F_{22}-F_{44}\right)^{2}=\frac{1}{4} F_{44}\left(4 F_{22}-F_{44}\right)=\frac{1}{4} F_{44}^{2} \delta$

where $\delta=4-\frac{F_{44}}{F_{22}}=4-\frac{\sigma_{2 t}^{*} \sigma_{2 c}^{*}}{\left(\tau_{23}^{*}\right)^{2}}$.

the conic section can be an ellipse, a parabola or a pair of hyperbolas, as sketched in Figure 4. Unless $\delta$ is positive, the locus in the $\sigma_{2}-\sigma_{3}$ plane will be open. It has been shown in [8] that there are real materials $\delta<0$, i.e. the conic section in the $\sigma_{2}-\sigma_{3}$ plane is open for such a materials. An open intersection naturally implies that the envelope will not be closed. Without meeting the closed failure envelope assumption, the original Tsai-Wu criterion will fall apart. It is again an example where a criterion cannot be taken for granted simply for its popularity. If the logic breaks down at any point, there will be consequences. The lack of trust in existing failure criteria from the practitioners is the ultimate penalty.

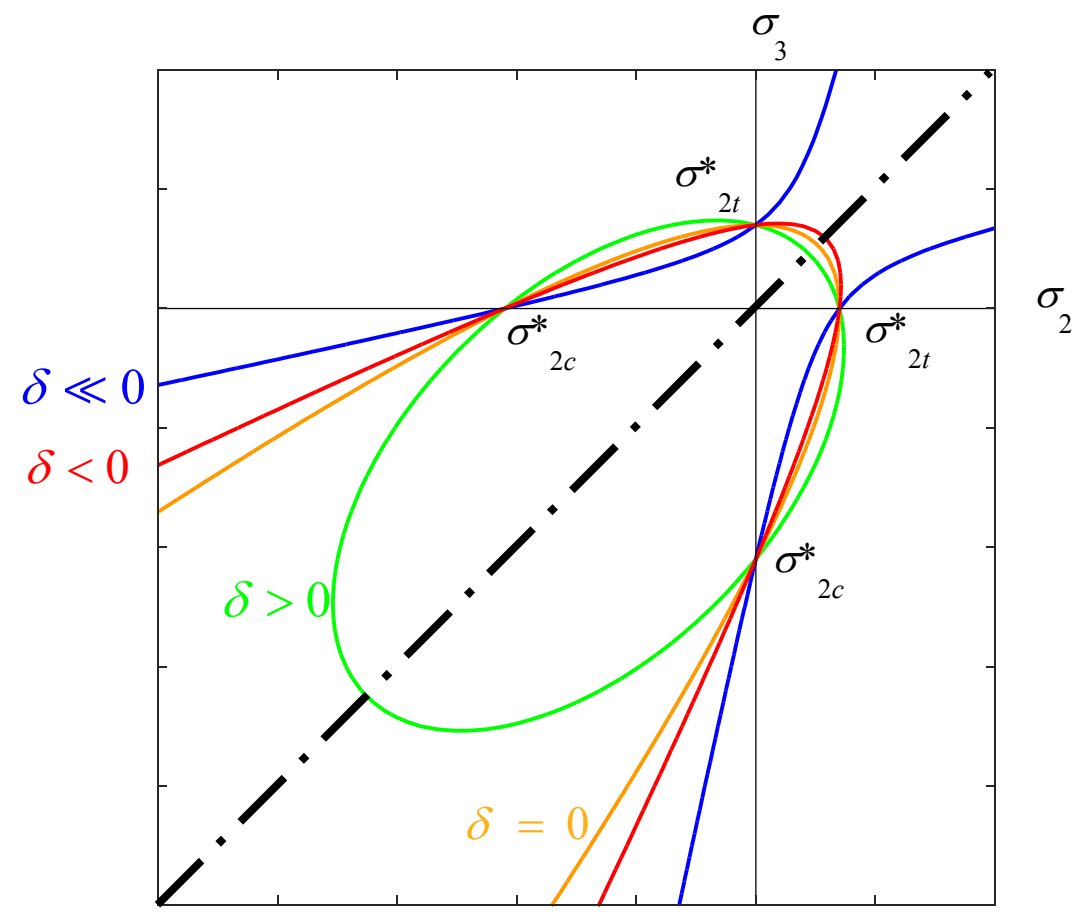

Figure 4 Failure loci in the plane direct stresses transverse to fibres 
On the specific topic of the Tsai-Wu criterion, readers are directed to a recent publication [8] where rational considerations have been made in order to respond to the irrational aspect as revealed above.

\section{The Hashin, the Puck and the LaRC04 criteria}

The Hashin criteria [9] were of epoch-making effect in the development of composites failure criteria by introducing systematic and rational measures in their formulation as opposed to purely empirical curve fitting. For instance, to construct the failure function, he started from a set of stress invariants, which form a set of irreducible invariant integrity bases in terms of rational mechanics [10]. The consequence of this was that objectivity of the theory had been implied without having to be imposed afterwards. He made the first attempt to separate different modes of failure by attempting a rational approach, i.e. exercising logical deduction to reach conclusions from a set of assumptions defined a priori, although only one of the key assumptions will be the focus of the present debate. He also took a number of rather arbitrary measures, in particular, when dropping some of the terms due to the lack of experimental means to determine them. There have been attempts to improve them, e.g., as recent as [11]. These will be considered in the present discussion as the derivatives of the basic assumptions in the Hashin criteria which, in the authors' opinion, are still too soon to be concerned. What should be examined first is the foundation, i.e. basic assumptions. Without solid foundation, any sophistication built in at the derivative end would be 'a house built on sand'.

Before addressing the basic assumption Hashin made, it is relevant to observe that a number of highly regarded failure criteria have emerged in recent years. Among them are the Puck criteria [12] and the so-called LaRC04 [13]. Without digging deep into these criteria, it can be noted that they share the following commonalities with high relevance to the present discussion in this paper.

1) They rest heavily on the Hashin criteria;

2) They all label themselves as physically based approaches.

To decode the label of 'physically based approach', it basically means that each criterion was constructed with due recognition of fracture surface. This would sound a formidable truth which could prevent such an approach from being challenged. However, this does not stop anyone taking a step forward by examining how the fracture surfaces were identified in these approaches. Then, one would find all of them point to the same basic assumption Hashin made, which effectively underpins the position of the Hashin criteria in the modern development of composites failure criteria. 
What Hashin assumed was that 'in the event that a failure plane can be identified, the failure is produced by the normal and shear stresses on that plane'. Furthermore, by defining fibre mode failure and matrix mode failure as fracture surface perpendicular or parallel to fibres, Hashin managed to separate failure modes into two mutually exclusive groups, fibre failure and matrix failure. Subsequent development in the Hashin criteria involved subdividing them into tensile and compressive modes before the failure function was simplified in each case.

In fact, the basic assumption Hashin made as quoted above was not new at all. It was one of the assumptions made in the Mohr criterion. However, Hashin did not make explicit reference to the Mohr criterion when he made this assumption (column 1 line 6 on p331 of [9]), which was an interesting subtlety. It was apparently not to cover up a plagiarism, as the Mohr failure theory was mentioned later on in his paper on a slightly different subject. When it was mentioned, it was enthusiastically endorsed as 'attractive because of its sound physical basis' (column 2 line 16 on p331 of [9]). Making such a claim usually suffices to convince most of the readers that the assumption Mohr made and Hashin inherited was 'physically sound'. Since then, the physical soundness of the assumptions has never been subject to serious challenge by the composites community. Followers of Hashin's criteria commonly tend to refer to their approaches as 'physically based' because of their use of a 'physically sound' assumption. Rather against the wind, the authors would like to reflect on this, inspired by the very spirit of physics throughout its history so far, which has been full of denial of established perceptions.

As the assumption has been so established, it will have to be addressed thoroughly. The authors will investigate how the basic assumption was misinterpreted in the Hashin criteria in a separate publication [14].

In this paper, attention will be turned to the origin of this assumption as a part of the Mohr criterion to strip off the formidable coating of 'physical soundness' before serious endeavours can be made into the development of composites failure criteria to bring the understanding closer to truth and hopefully meet the industrial needs in long run.

\section{The Mohr criterion}

The Mohr criterion was proposed for isotropic materials. It took advantage of graphic representation of 3D stress states in terms of Mohr's circles, defined by three principal stresses, as illustrated in Figure 5. For the ease of reference, the outmost Mohr's circle constructed from the $1^{\text {st }}$ and the $3^{\text {rd }}$ principal stresses will be referred to as the major Mohr's circle of the stress state concerned. The Mohr criterion can then be formulated and presented in a very rational manner in 
the sense that it can be deduced from two assumptions: (A) failure is dictated by the stresses (one direct and one shear) exposed on the fracture surface, and (B) the existence of a failure envelope, sketched in Figure 6, as the curve which circumscribes all major Mohr's circles for all possible stress states for a given material. A natural consequence of assumption (A) will be that the intermediate principal stress $\sigma_{2}$ does not contribute to failure, since the major Mohr's circle has nothing to do with $\sigma_{2}$.

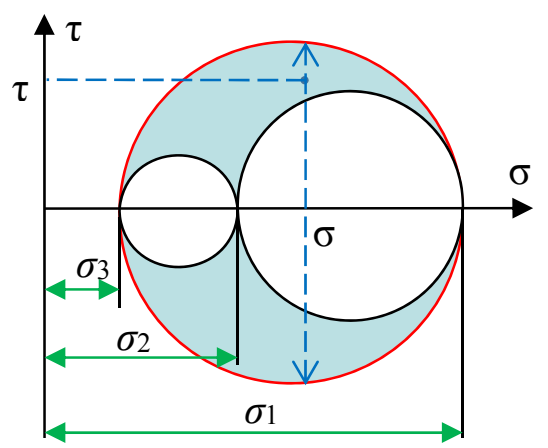

Figure 5 Mohr's circles for 3D stress state

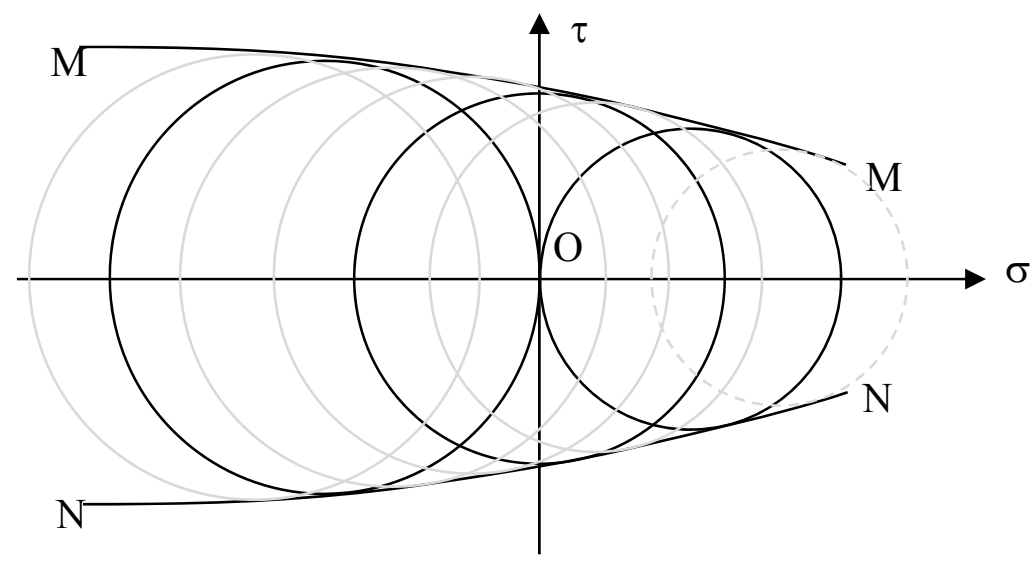

Figure 6 Failure envelope of the Mohr criterion

With the two assumptions, the Mohr criterion states that for any given stress state, if its major Mohr's circle falls within the failure envelope, it is safe, and otherwise failure is expected. As it is, the Mohr criterion is non-phenomenological, i.e. it does not predict failure mode and does not rely on the knowledge of the failure surface, either, in order to predict failure. Although it employs the concept of stresses on the fracture surface, it does not identify the orientation of the fracture surface.

The Mohr criterion as presented above is quite rational as everything has been deduced from two assumptions made. However, it should be clarified that these two assumptions remain assumptions, no matter how rational the subsequent deduction is in the formulation of the criterion. None of the assumptions should be taken for granted. In fact, the first assumption would fall apart if one found two stress states of identical $\sigma_{1}$ and $\sigma_{3}$ but different $\sigma_{2}$ fail at different load levels. This would also 
dismiss the existence of the failure envelope as the second assumption. Experimental data suggesting the observable differences $\sigma_{2}$ made had been produced for some materials as early as 1911 [15], shortly after Mohr published his theory in 1900 [16].

Readers are reminded that the von Mises criterion accounts for $\sigma_{2}$. At the same $\sigma_{1}$ and $\sigma_{3}$, different $\sigma_{2}$ leads to different failure load. The von Mises criterion is one of the most popular criteria, if not the most popular one. The materials that comply with it defy the Mohr criterion. One might argue that the von Mises criterion is meant to be for ductile materials, like the Tresca criterion. In fact, the Mohr criterion can be considered as a counterpart of the Tresca criterion for brittle materials of which the tensile and compressive strengths are significantly different. In much the same way, the Raghava criterion [17] can be considered the counterpart of the von Mises criterion for brittle materials. One can then argue that the materials complying with the Raghava criterion will not perform well with the Mohr criterion. A similar discussion on the performances of the Tresca criterion and the von Mises criterion was made in early days of the development of the theory of plasticity [18]. To relate the relevant criteria employed in the discussion above, they have been listed in Table 1 for the readers' information.

Table 1 Comparison between relevant failure criteria

\begin{tabular}{cll}
\hline Material type & \multicolumn{2}{c}{ Stresses employed } \\
\cline { 2 - 3 } & $1^{\text {st }}$ and $3^{\text {rd }}$ principal stresses & All three principal stresses \\
\hline Ductile & Tresca & Von Mises \\
Brittle & Mohr & Raghava at al. [17] \\
\hline
\end{tabular}

The argument here is to deliver the message as follows. The Mohr criterion is a good and rational criterion, but not without backlashes, nor universally applicable, even for brittle isotropic materials. As the Mohr criterion is rather rational, any insufficiency must have rested in the assumptions. In other words, even for brittle isotropic materials, those introduced as the underlying assumptions for the Mohr criterion may not be trusted blindly. In other words, the Mohr criterion is not as physically sound as one would like, not even for the class of materials which suit the criterion the most. Claiming the physical soundness of any theories based on the Mohr criterion is a gross overstatement of the fact.

A more significant unjustified leap of faith was its application to composites which are anisotropic. The Mohr criterion was based on the Mohr's circles for 3D stresses by making use of the principal stresses. In composites, the stresses are not principal stresses in the same sense as those employed in the Mohr criterion or Mohr's circle. They are stresses in the material's principal axes, sometimes 
mistakenly called the principal stresses. A simple disparity for readers to appreciate is the fact that stresses in material's principal axes involve non-vanishing shear components. The reliance on principal stresses has deprived the applicability of the Mohr criterion to composites. Any employment of it without due diligence amounts to nothing but an abuse.

\section{$7 \quad$ Conclusions}

A critical assessment of the criteria that are widely used nowadays to define failure in composites have been conducted. Three typical groups of criteria have been appraised, namely, those not involving interactions between the stress or strain components; criteria accounting for interactions between the stress components, and the criteria dependent failure modes. It has been demonstrated that all of them suffer from drawbacks one way or another, all of a fundamental nature but not documented in the literature.

Specifically, the lack of objectivity was revealed for the maximum stress criterion. Its counterpart, the maximum strain criterion, was constructed using the strength properties obtained under uniaxial stress conditions but expressed in terms of strains at failure. This could potentially result in confusions and inconsistencies, in particular, in failure mode prediction.

The most popular failure criterion incorporating stress interactions is the Tsai-Wu failure criterion, for which it has been shown explicitly that there are materials that simply defy one of the major underlying assumptions of this failure theory.

A significant number of modern failure criteria for composites was formulated based on the failure modes. They have been labelled with an overstated tag of being 'physically based' by employing an assumptions introduced as a part of the Mohr criterion, whilst the universal applicability of Mohr's assumption remains questionable even for brittle isotropic materials, let alone composites.

With the fundamental shortcomings of the popular composite failure theories thus revealed, the lack of rationality in the formulation of composite failure criteria can be seen as an acute symptom to be addressed in order to progress in this front of scientific endeavour before any further sophistications to be added to the derivative ends and existing theories.

\section{References}

[1] CMH-17, 2012. Composite Materials Handbooks, Vol. 3 Polymer matrix composites materials usage, design, and analysis, SAE International, Wichita State University, USA 
[2] Federal Aviation Administration Advisory Circular, 2010. Composite Aircraft Structure, AC No:20-107B, Aug 2010, Change 1

[3] E. Sitnikova, S. Li, D. Li, X. Yi, 2017. Subtle features of delamination in cross-ply laminates due to low speed impact, Composites Science and Technology, 149:149-158

[4] M.J. Hinton, P.D. Soden, and A.S. Kaddour, 1998, 2002 and 2004. "Failure Criteria in Fibre-Reinforced-Polymer Composites", Composites Science and Technology, Part A, 58(7); Part B, 62(12-13) and Part C, 64(3-4)

[5] A.S. Kaddour, and M.J. Hinton, 2012 and 2013. "Evaluation of Theories for Predicting Failure in Polymer Composite Laminates under 3-D States of Stress", Journal of Composite Materials, Part A, 46(19-20) and Part B, 47(6-7)

[6] A.S. Kaddour, M.J. Hinton, P.A. Smith, and S. Li, 2013. "Benchmarking of matrix cracking, damage and failure models for composites: Comparison between theories", Part A, Journal of Composite Materials, 47(20-21)

[7] S. Timoshenko, 1957. Strength of Materials Part II, Advanced Theory and Problems, $3^{\text {rd }}$ edn., D. Van Nostrand Company, Inc., Amsterdam

[8] S. Li, E. Sitnikova, Y. Liang, A.-S. Kaddour, 2017. The Tsai-Wu failure criterion rationalised in the context of UD composites, Composites Part A, 102:207-217

[9] Z. Hashin, 1980. Failure Criteria for Unidirectional Fiber Composites, Journal of Applied Mechanics, 47:329-334

[10] A.C. Pipkin and R.S. Rivlin, 1959, The foundation of constitutive equations in continuum physics, Arch. Rational Mech. Anal., 4:129-144

[11] J. Gu, and P. Chen, 2017. Some modifications of Hashin's failure criteria for unidirectional composite materials, Composite Structures, 182:143-152

[12] M. Knops, 2008. Analysis of Failure in Fiber Polymer Laminates, The Theory of Alfred Puck, Springer, Berlin

[13] S.T. Pinho, C.G. Dávila, L. Iannucci and P. Robinson, 2005. Failure models and criteria for FRP under in-plane or three-dimensional stress states including shear non-linearity, NASA/TM-2005-213530, NASA Langley Research Center, Hampton, VA 23681, USA

[14] S. Li and E. Sitnikova, 2017. A critical examination of the underlying assumptions in the Hashin criteria, in preparation

[15] T. von Karman, 1911, Festigkeitsversuche unter all seitigem Druck [Strength tests under pressure from all sides], Z. Verein Deut. Ingr. 55, 1749-1759 (In German).

[16] O. Mohr, 1900. Welche Umstiinde bedingen die Elastizitatsgrenze und den Bruch eines Materiales, Zeitschrift des Vereines Deutscher Ingenieure, 44:1524-1530 (In German)

[17] R. Raghava, R.M. Caddell and G.S.Y. Yeh, 1973. The macroscopic yield behaviour of polymers, J. Mater. Sci., 8:225

[18] R. Hill, 1950. The mathematical theory of plasticity, Oxford University Press, Inc., Oxford 